\title{
Direct Electroanalytical Determination of Fluvastatin in a Pharmaceutical Dosage Form: Batch and Flow Analysis
}

\author{
Marta M. P. S. Neves, Henri P. A. Nouws, and \\ Cristina Delerue-Matos
}

\begin{abstract}
The reduction of luvastatin (FLV) at a hanging mercury-drop electrode (HMDE) was studied by square-wave adsorptive-stripping voltammetry (SWAdSV). FLV can be accumulated and reduced at the electrode, with a maximum peak current intensity at a potential of approximately $-1.26 \mathrm{~V}$ vs. $\mathrm{AgCl}=\mathrm{Ag}$, in an aqueous electrolyte solution of $\mathrm{pH} 5.25$. The method shows lin- earity between peak current intensity and FLV concentration between $1.0 \times 10^{-8}$ and $2.7 \times 10^{-6} \mathrm{~mol} \mathrm{~L}^{-1}$. Limits of detection (LOD) and quantification (LOQ) were found to be $9.9 \times 10^{-9}$ mol L ${ }^{-1}$ and $3.3 \times 10^{-8} \mathrm{~mol} \mathrm{~L}^{-1}$, respectively.

Furthermore, FLV oxidation at a glassy carbon electrode surface was used for its hydrodynamic monitoring by amperometric detection in a flow-injection system. The amperometric signal was linear with FLV concentration over the range $1.0 \times$ $10^{-6}$ to $1.0 \times 10^{-5} \mathrm{~mol} \mathrm{~L}^{-1}$, with an LOD of $2.4 \times 10^{-7} \mathrm{~mol} \mathrm{~L}^{-1}$ and an LOQ of $8.0 \times 10^{-7} \mathrm{~mol} \mathrm{~L}{ }^{-1}$. A sample rate of 50 injections per hour was achieved.
\end{abstract}

\section{Keywords}

adsorptive-stripping voltammetry, amperometric detection, flow injection analysis, Fluvastatin, hanging mercury drop electrode, pharmaceutical analysis

\section{INTRODUCTION}

Nowadays, statins are the most effective antihypercholesterolemic agents used worldwide (Paoletti, Corsini, and Bellosta 2002; Schachter 2004). These compounds reduce levels of circulating atherogenic lipoproteins by competitive inhibition of HMG-CoA redutase, the rate-limiting enzyme in the synthesis of cholesterol (Endo 2004). Fluvastatin (FLV, Fig. 1) is one of these lipid-lowering drugs and the first entirely synthetic statin.

No official quantification methods are reported in the various pharmacopoeias. The majority of the methods proposed for the determination of FLV are based on separation methods and are applied in the analysis of serum or plasma samples. In Table 1, a summary of these methods is indicated, including some performance parameters. Several authors propose the analysis of the racemates of FLV (Toreson and Eriksson 1996, 1997; Lanchote et al. 2001; Di Pietro et al. 2006), and in one case several metabolic products were also analysed (Nakashima et al. 2001).

Besides the methods included in Table 1, two electroanalytical procedures are also described (Ozkan and Uslu 2002; Dogan et al. 2007), which 
are based on the electro-oxidative behaviour of FLV in aqueous solution using either a glassy-carbon (Ozkan and Uslu 2002) or a boron-doped diamond (Dogan et al. 2007) working electrode. The methods showed similar linear ranges $\left(10^{-4}\right.$ to $\left.10^{-6} \mathrm{~mol} \mathrm{~L}^{-1}\right)$ with limits of detection at submicromolar levels and were applied to pharmaceutical and spiked human serum samples. Until now, no determination of FLV based on its electroreduction has been described, and also no hydrodynamic monitoring using electrochemical detection has been reported.

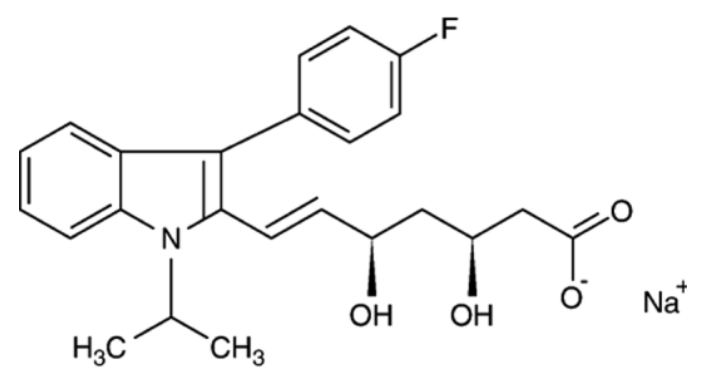

Figure 1. Chemical structure of Fluvastatin. 
Table 1. Analytical methods developed for the analysis of fluvastatin in serum= plasma and $=$ or pharmaceutical products

\begin{tabular}{|c|c|c|c|c|}
\hline Method $^{a}$ & Detection $^{a}$ & $\begin{array}{l}\text { Linear range } \\
\left(\text { nmol L }{ }^{-1}\right)\end{array}$ & $\begin{array}{c}\text { LOQ } \\
\left(\mathrm{nmol} \mathrm{L}^{-1}\right)\end{array}$ & Reference \\
\hline $\mathrm{GC}$ & MS & $5-1247$ & 4.9 & Leis and Windischhofer 2005 \\
\hline \multirow[t]{8}{*}{$\mathrm{LC}$} & FL & $1-2436$ & - & Al-Rawithi et al. 2003 \\
\hline & & $1-244$ & 1.2 & Um et al. 2006 \\
\hline & & $1-5000$ & 0.5 & Toreson and Eriksson 1996 \\
\hline & & $0.5-3200$ & 0.5 & Toreson and Eriksson 1997 \\
\hline & & $2-1523$ & 1.8 & Lanchote et al. 2001 \\
\hline & MS & $4-2436$ & 3.7 & Di Pietro et al. 2006 \\
\hline & & $5-1218$ & 4.9 & Nirogi et al. 2006 \\
\hline & UV & $24-2436$ & 24 & Nakashima et al. 2001 \\
\hline $\mathrm{CE}$ & UV & $(1.0-5.2) \times 10^{4}$ & 2900 & Dogrukol-Ak et al. 2001 \\
\hline UV & & $(2.4-6.8) \times 10^{4}$ & - & Erk 2002 \\
\hline
\end{tabular}

${ }^{a} \mathrm{GC}$, gas chromatography; LC, liquid chromatography; CE, capillary electrophoresis; UV, ultraviolet spectrophotometry; MS, mass spectrometric; FL, fluorimetric.

In the present study, two methodologies for the quantification of FLV in a pharmaceutical product are proposed. Both methods are based on the electrochemical behavior of FLV. Square-wave adsorptivestripping voltammetry (SWAdSV) was used under batch conditions, and amperometric detection was included in a flow injection analysis system (FIA-EC).

\section{EXPERIMENTAL}

\section{Apparatus}

Voltammetric measurements, using SWV and SWAdSV, were performed using an Autolab PGSTAT12 (Metrohm-EcoChemie) potentiostat, controlled by a PC running GPES 4.9 software (Metrohm-EcoChemie), and a Metrohm 663 VA stand containing a three-electrode cell (all Metrohm). This voltammetric cell consisted of a multimode mercury working electrode, used in the hanging mercury-drop position (HMDE), an $\mathrm{Ag}=\mathrm{AgCl}=\mathrm{KCl} 3 \mathrm{~mol} \mathrm{~L}^{-1}$ reference electrode, and a glassy carbon auxiliary electrode.

In the single-manifold FIA system, solutions were transported to the amperometric detection cell by a Gilson Miniplus 3 peristaltic pump, using PTFE tubing (i.d. $0.8 \mathrm{~mm})$. Samples and standards $\left(V_{\text {inj }} 1 / 4238.6 \mathrm{~mL}\right)$ 
were introduced into the carrier stream through a six-port Rheodyne 5041 injection valve. Amperometric detection was carried out in a Metrohm 656 wall-jet cell coupled to a Metrohm 641 VA-detector. Working, reference, and auxiliary electrodes were glassy carbon, $\mathrm{Ag}=\mathrm{AgCl}=\mathrm{KCl} 3 \mathrm{~mol}$ $\mathrm{L}^{-1}$, and gold, respectively. The output signals were recorded on a Kipp \& Zonnen BD 111 strip chart recorder. When required, the glassy carbon electrode was cleaned mechanically by polishing its surface with $\mathrm{Al}_{2} \mathrm{O}_{3}$ with a particle size of $0.015 \mathrm{~mm}$ (BDH Chemicals) on a wet polishing cloth. Flow injection signals were evaluated in terms of peak height $(\mathrm{cm})$ and afterward converted into current $(\mathrm{mA})$.

\section{Reagents and Solutions}

A FLV sodium standard was purchased from Calbiochem and used without further purification. Stock solutions of FLV $\left(1.0 \times 10^{-3} \mathrm{~mol} \mathrm{~L}^{-1}\right)$ were prepared in water and stored in the dark at $4^{\circ} \mathrm{C}$. All chemicals used were of analytical reagent-grade quality. Deionized water (conductivity $<0.1 \mathrm{mS} \mathrm{cm}^{-1}$ ) was obtained from a Millipore Simplicity-185 system.

For SWAdSV, working solutions were diluted with water and electrolyte solution to furnish the desired concentration. Two different buffers were tested in electrolyte $\mathrm{pH}$ optimization, a Britton-Robinson buffer (pH 1.9 - 11.8) (Fern' andezand Martin 1977) and an acetate (pH 4.5 - 6.0; $0.2 \mathrm{~mol} \mathrm{~L}^{-1}$ ) buffer. For FIA, solutions were prepared by diluting the stock solution with electrolyte to furnish the desired concentrations and were injected into the carrier stream (Britton-Robinson buffer, $\mathrm{pH}$ 10.0).

\section{Procedures}

General Procedure

In SWAdSV, the supporting electrolyte $(10.00 \mathrm{~mL})$ was purged with oxygen-free nitrogen for $300 \mathrm{~s}$ and for $3 \mathrm{~s}$ in subsequent scans. After the oxygen removal, accumulation of FLV at the electrode surface was performed, and a square-wave potential scan in the negative direction was applied.

Using FIA-EC, the background current (baseline) was recorded continuously while the electrolyte flowed through the detection cell.

\section{Validation Procedure}

Several operational parameters of both methods were studied and optimized for FLV determination. Using the optimal experimental 
conditions, validation of the methods was performed via evaluation of the linear range, limit of detection (LOD), limit of quantification (LOQ), repeatability, intermediate precision, accuracy, and selectivity.

Repeatability and intermediate precision were assessed at three concentrations. For repeatability assays, five measurements of each solution were made in a short period of time; to determine intermediate precision, the solutions were analyzed for 3 consecutive days. For the SWAdSV method, concentrations of $1.0 \times 10^{-7} \mathrm{~mol} \mathrm{~L}^{-1}, 1.3 \times 10^{-6} \mathrm{~mol}$ mol L ${ }^{-1}$, and $2.5 \times 10^{-6} \mathrm{~mol} \mathrm{~L}^{-1}$ were used. The precision of the FIAEC method was evaluated by use of $2.0 \times 10^{-6} \mathrm{~mol} \mathrm{~L}^{-1}, 5.0 \times 10^{-6} \mathrm{~mol}$ $\mathrm{L}^{-1}$, and $8.0 \times 10^{-6} \mathrm{~mol} \mathrm{~L}^{-1} \mathrm{FLV}$ solutions. To assess the accuracy and selectivity, known amounts of the pure drug were added to solutions of the pharmaceutical product at three concentration levels. In the evaluation of the SWAdSV method, a solution of the pharmaceutical containing $1.0 \times 10^{-7} \mathrm{~mol} \mathrm{~L}^{-1}$ FLV was spiked with FLV standard to furnish final concentrations of $5.0 \times 10^{-7} \mathrm{~mol} \mathrm{~L}^{-1}, 1.5 \times 10^{-6} \mathrm{~mol} \mathrm{~L}^{-1}$, and $2.5 \times 10^{-6} \mathrm{~mol} \mathrm{~L}^{-1}$. For the flow-injection method, FLV standard concentration levels of $1.0 \times 10^{-6} \mathrm{~mol} \mathrm{~L}^{-1}, 4.0 \times 10^{-6} \mathrm{~mol} \mathrm{~L}^{-1}$ and $7.0 \times 10^{-6} \mathrm{~mol} \mathrm{L^{-1 }}$ were added to volumetric flasks containing $1.0 \times 10^{-6} \mathrm{~mol} \mathrm{~L}^{-1}$ of FLV.

\section{Pharmaceuticals Analysis}

The methods were used to quantify FLV sodium in a pharmaceutical dosage form (Lescol-Novartis), containing $20 \mathrm{mg}$ of FLV per capsule. Lescol contains the excipients magnesium stearate, gelatine, microcrystalline cellulose, pregelatinized starch, red iron oxide, sodium lauryl sulphate, talc, titanium dioxide, and yellow iron oxide, among others.

The content of one capsule was dissolved in $500.0 \mathrm{~mL}$ of water by sonication for $30 \mathrm{~min}$ (solution A).

For batch analysis, the resulting solution was diluted 100-fold with water, and an adequate volume of this diluted solution was added to $10.00 \mathrm{~mL}$ of electrolyte contained in a voltammetric cell to achieve an FLV concentration of $1.0 \times 10^{-7} \mathrm{~mol} \mathrm{~L}^{-1}$. Using the standard addition method, increasing quantities of FLV standard between 0 and $1.3 \times 10^{-7} \mathrm{~mol} \mathrm{~L}^{-1}$ were added to the solution in the voltammetric cell. This procedure was repeated in triplicate. The use of the standard addition method and the large dilution make the filtration of solution A unnecessary.

For hydrodynamic detection, an accurate volume of solution A, corresponding to a final FLV concentration of $5.0 \times 10^{-6} \mathrm{~mol} \mathrm{~L}^{-1}$, was transferred to a $10.00-\mathrm{mL}$ volumetric flask. The volume was completed 
with Britton-Robinson buffer, $\mathrm{pH}$ 10.0. The quantification was carried by interpolation of a calibration curve $\left(1.0 \times 10^{-6} \mathrm{~mol} \mathrm{~L}^{-1}\right.$ to $1.0 \times$ $10^{-5} \mathrm{~mol} \mathrm{~L}^{-1}$ ). This procedure was repeated in triplicate. Again, no additional treatment of the samples was required.

\section{RESULTS AND DISCUSSION}

\section{Square-Wave Adsorptive-Stripping Voltammetry (SWAdSV)}

The electrochemical behaviour of FLV was studied at an HMDE under batch conditions by SWV and SWAdSV. FLV was reduced in aqueous medium at the HMDE over a wide $\mathrm{pH}$ range $(3.1$ - 11.8, Britton-Robinson buffer). The analytical signal increased with increasing electrolyte $\mathrm{pH}$ up to $\mathrm{pH}$ 5.25. After comparison with an acetate buffer ( $\mathrm{pH}$ 4.5-6.0), results showed that the highest and best-defined peak was obtained at $\mathrm{pH} 5.25$, which was chosen as supporting electrolyte in all further experiments. At this $\mathrm{pH}$ value, FLV presented a reduction peak at approximately $-1.26 \mathrm{~V}$, which increased with increasing accumulation time $\left(t_{\text {acc }}\right)$. For a $1.0 \times 10^{-6} \mathrm{~mol} \mathrm{~L}^{-1} \mathrm{FLV}$ solution, this increase is significant for up to approximately $130 \mathrm{~s}$ at an optimized accumulation potential $\left(E_{\text {acc }}\right)$ of $-0.3 \mathrm{~V}$. For longer accumulation times, the increase of $i_{\mathrm{p}}$ levels off, possibly due to the saturation coverage of the drop and=or competitive adsorption. The best compromise between $i_{\mathrm{p}}$ and analysis time was achieved with a $t_{\mathrm{acc}}$ of $30 \mathrm{~s}$. The square-wave potential scan was optimized regarding frequency $(f)$, pulse step $\left(\mathrm{D} E_{\mathrm{s}}\right)$, and pulse amplitude $\left(\mathrm{D} E_{\mathrm{p}}\right)$; these parameters are interrelated and have a combined effect on the analytical signal. Different values for $f(50-1000 \mathrm{~Hz}), \mathrm{D} E_{\mathrm{s}}$ $(1-5 \mathrm{mV})$, and $\mathrm{D} E_{\mathrm{p}}(10-50 \mathrm{mV})$ were studied. The best relation among peak current intensity, peak definition, and repeatability was achieved with the follow conditions: $f^{1 / 4} 500 \mathrm{~Hz}, \mathrm{D} E_{\mathrm{s}}{ }^{1 / 4} 5 \mathrm{mV}$, and $\mathrm{D} E_{\mathrm{p}} 1 / 450 \mathrm{mV}$. These conditions were used in all subsequent studies.

A linear relationship between stripping peak current intensity and FLV concentration was obtained for the interval between $1.0 \times 10^{-8}$ and $2.7 \times 10^{-6} \mathrm{~mol} \mathrm{~L}^{-1}$. Some voltammograms of FLV solutions in the linear range are shown in Fig. 2. In Table 2, several characteristics of the calibration plot, as well as the LOD and LOQ calculated using this plot, are indicated.

Results of precision and accuracy studies, presented in Table 3, confirm that the method is precise, accurate, and selective for the analysis of FLV.

The method was applied to the quantification of FLV in Lescol. Analyses were preformed using five standard additions between 0 and $1.3 \times 10^{-7} \mathrm{~mol} \mathrm{~L}^{-1}$, obtaining a result of $20.04 \pm 0.05 \mathrm{mg}=$ capsule $\left(\mathrm{n}^{1 / 4} 3\right)$. 


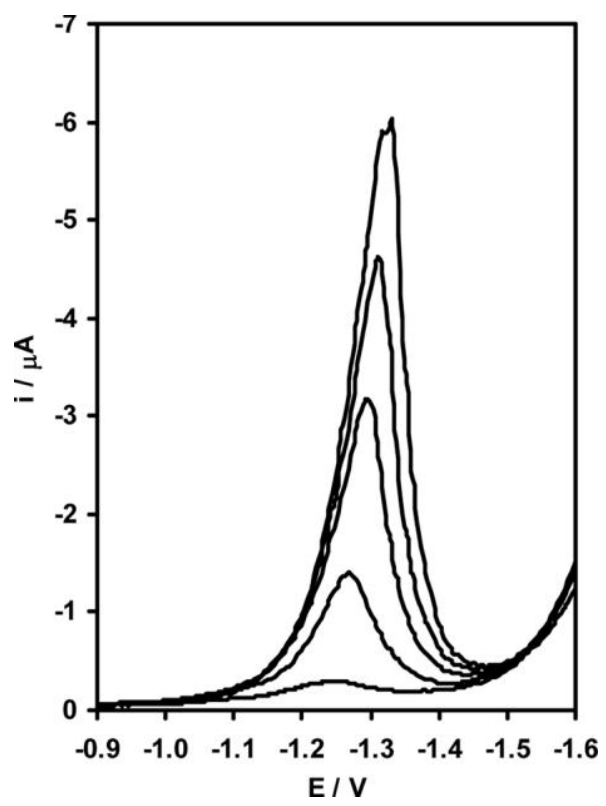

Figure 2. SWAdSV voltammograms of FLV solutions in the linear range. [FLV] $(\mathrm{mol} \mathrm{L}-1): 1.0 \times 10^{-8}, 5.0 \times 10^{-7}, 1.3 \times 10^{-6}, 1.9 \times 10^{-6}$, and $2.7 \times 10^{-6}$.

Flow-Injection Analysis with Amperometric Detection (FIA-EC)

An FIA-EC method for FLV determination in a pharmaceutical product was also developed. The method is based on FLV oxidation in a pH 10.0

Table 2. Analytical data from the calibration plot in the determination of FLV by SWAdSV and FIA-EC

\begin{tabular}{|c|c|c|}
\hline Parameter & SWAdSV & FIA-EC \\
\hline Linear range $\left(\mathrm{mol} \mathrm{L}^{-1}\right)$ & $\begin{array}{c}1.0 \times 10^{-8} \text { to } \\
2.7 \times 10^{-6}\end{array}$ & $\begin{array}{c}1.0 \times 10^{-6} \text { to } \\
1.0 \times 10^{-5}\end{array}$ \\
\hline$n$ & 13 & 6 \\
\hline Correlation coefficient & 0.999 & 0.999 \\
\hline $\begin{array}{l}\text { Slope of calibration } \\
\left.\text { plot }(\mathrm{A} \text { mol L L })^{-1}\right)^{-1}\end{array}$ & 2.460 & 0.0620 \\
\hline Standard error slope & 0.004 & 0.0008 \\
\hline Intercept (nA) & 255 & -2.90 \\
\hline Standard error intercept & 8 & 243 \\
\hline $\mathrm{LOD}\left(\mathrm{nmol} \mathrm{L}{ }^{-1}\right)$ & 9.9 & 5.02 \\
\hline LOQ (nmol L ${ }^{-1}$ ) & 33 & 810 \\
\hline
\end{tabular}


Table 3. Results from evaluation of the precision and accuracy $^{a}$

\begin{tabular}{llc}
\hline Property & SWAdSV & FIA-EC \\
\hline Repeatability (RSD \%) & $0.61(0.1)$ & $5.1(2.0)$ \\
& $1.1(1.3)$ & $4.3(5.0)$ \\
Intermediate precision (RSD \%) & $0.77(2.5)$ & $1.4(8.0)$ \\
& $5.0(0.1)$ & $7.1(2.0)$ \\
Recovery (\%) & $4.1(1.3)$ & $2.0(5.0)$ \\
& $0.51(2.5)$ & $3.9(8.0)$ \\
& $96.8 \pm 2.4(0.4)$ & $99.5 \pm 6.1(1.0)$ \\
& $98.0 \pm 1.3(1.4)$ & $99.0 \pm 2.4(4.0)$ \\
& $103.8 \pm 1.4(2.4)$ & $100.1 \pm 2.8(7.0)$ \\
\hline
\end{tabular}

${ }^{a}$ Concentration in $\mathrm{mmol}=\mathrm{L}$ are given in parentheses.

Britton-Robinson buffer described in a previous work (Ozkan and Uslu 2002). The oxidation current was monitored at a glassy carbon working electrode, and several experimental conditions were optimized to achieve the best relation among repeatability, reproducibility, sensitivity, sampling rate, and reagent consumption. The parameters optimized were the working electrode potential, the injection volume, the length of reactor, and the flow rate. This led to the following optimal conditions: $E^{1 / 4} 1.0 \mathrm{~V} ; V_{\text {inj }} 1 / 4238.6 \mathrm{~mL}$; reactor length $1 / 418 \mathrm{~cm}$; and flow rate $1 / 4$ $1.6 \mathrm{~mL}=\min$, achieving a sample rate of up to 50 injections per hour.

The frequently reported adsorption of the oxidation products on the electrode surface was also observed in this study. This adsorption leads to a lack of available surface and consequently a decrease of the analytical signal; therefore cleaning of the electrode surface was necessary. However, flow systems minimize this problem because of the constant passage of the carrier stream, which helps to keep the electrode surface clean. In the present work, the electrode was polished only at the beginning of every working period.

Under the optimal conditions, the amperometric signal is linear for FLV concentrations between $1.0 \times 10^{-6} \mathrm{~mol} \mathrm{~L}^{-1}$ and $1.0 \times 10^{-5} \mathrm{~mol}$ $\mathrm{L}^{-1}$, with an LOD of $2.4 \times 10^{-7} \mathrm{~mol} \mathrm{~L}^{-1}$ and an LOQ of $8.0 \times 10^{-7} \mathrm{~mol}$ mol L ${ }^{-1}$. Calibration characteristics and the validation data are given in Tables 2 and 3 and indicate that the method is precise, accurate, and selective for FLV analysis in the tested pharmaceutical product. In Figure 3, a fiagram of several FLV solutions with concentrations in the linear range is shown.

The result of the quantification of FLV in the pharmaceutical product Lescol using the developed method was $20.3 \pm 0.4 \mathrm{mg}=$ capsule $(n 1 / 43)$. 


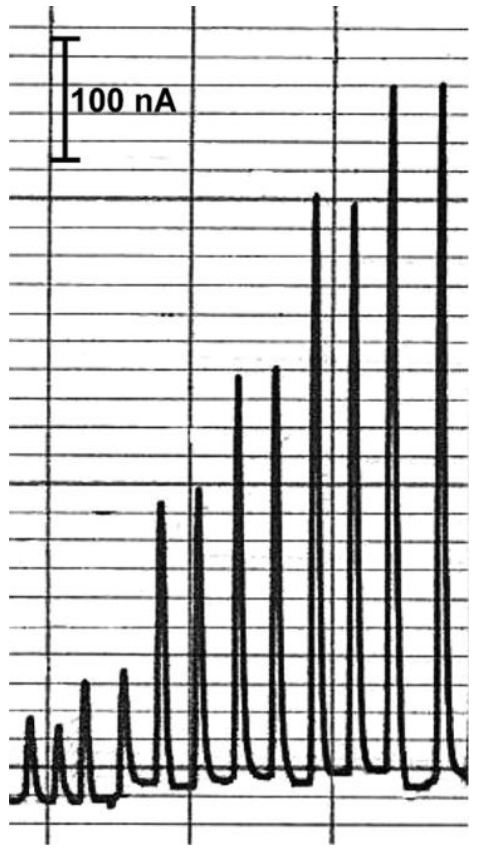

Figure 3. Fiagram of various FLV solutions in the linear range. [FLV] (mmol $\left.\mathrm{L}^{-1}\right):$ 1.0, 2.0, 4.0, 6.0, 8.0, and 10 .

\section{CONCLUSION}

Based on FLV' s electrochemical behavior, two easy and fast analytical methods were developed for its determination in a pharmaceutical product. SWAdSV and FIA-EC were successfully applied without interferences from the excipients. Based on the linear ranges and limits of detection, both electroanalytical methodologies could be useful for the quantification of FLV in the tested product. Although the SWAdSV method presents higher sensitivity and a lower LOD, which can be useful for clinical analysis, the FIA-EC method presented a higher sample throughput, and its ease of automation could allow its adoption in routine pharmaceutical quality control.

\section{REFERENCES}

Al-Rawithi, S., Hussein, R.F., and Alzahrani, A. 2003. Sensitive assay for the determination of fluvastatin in plasma utilizing high-performance liquid chromatography with fluorescence detection. Ther. Drug Monit., 25(1): 88 - 92. 
Di Pietro, G., Coelho, E.B., Geleilete, T.M., Marques, M.P., and Lanchote, V.L. 2006. Chiral evaluation of fluvastatin in human plasma by high-performance liquid chromatography electrospray mass spectrometry. J. Chromatogr. B, 832(2): $256-261$.

Dogan, B., Tuncel, S., Uslu, B., and Ozkan, S.A. 2007. Selective electrochemical behavior of highly conductive boron-doped diamond electrodes for fluvastatin sodium oxidation. Diam. Relat. Mat., 16(9): 1695 - 1704.

Dogrukol-Ak, D., Kircali, K., Tuncel, M., and Aboul-Enein, H.Y. 2001. Validated analysis of fluvastatin in a pharmaceutical capsule formulation and serum by capillary electrophoresis. Biomed. Chromatogr., 15(6): $389-392$.

Endo, A. 2004. The discovery and development of HMG-CoA reductase inhibitors. Atheroscler. Suppl., 5(3): $67-80$.

Erk, N. 2002. Rapid spectrophotometric method for quantitative determination of simvastatin and fluvastatin in human serum and pharmaceutical formulations. Pharmazie, 57(12): 817 - 819.

Fern' andez, C.M. and Martin, V.C. 1977. Talanta, 24: 747 - 748.

Lanchote, V.L., Rocha, A., de Albuquerque, F.U.V., Coelho, E.B., and Bonato, P.S. 2001. Stereoselective analysis of fluvastatin in human plasma for pharmacokinetic studies. J. Chromatogr. B, 765(1): 81 - 88.

Leis, H.J. and Windischhofer, W. 2005. Quantitative determination of fluvastatin in human plasma by gas chromatography=negative ion chemical ionization mass spectrometry using [O-18(2)]-fluvastatin as an internal standard. Rapid Commun. Mass Spectrom., 19(2): 128 - 132.

Nakashima, A., Saxer, C., Niina, M., Masuda, N., Iwasaki, K., and Furukawa, K. 2001. Determination of fluvastatin and its five metabolites in human plasma using simple gradient reversed-phase high-performance liquid chromatography with ultraviolet detection. J. Chromatogr. B, 760(1): 17 - 25.

Nirogi, R.V.S., Kandikere, V.N., Shrivastava, W., Mudigonda, K., and Datla, P.V. 2006. Liquid chromatography=negative ion electrospray tandem mass spectrometry method for the quantification of fluvastatin in human plasma: Validation and its application to pharmacokinetic studies. Rapid Commun. Mass Spectrom., 20(8): 1225 - 1230.

Ozkan, S.A. and Uslu, B. 2002. Electrochemical study of fluvastatin sodium analytical application to pharmaceutical dosage forms, human serum, and simulated gastric juice. Anal. Bioanal. Chem., 372(4): 582 - 586.

Paoletti, R., Corsini, A., and Bellosta, S. 2002. Pharmacological interactions of statins. Atheroscler. Suppl., 3(1):35 - 40.

Schachter, M. 2004. Chemical, pharmacokinetic, and pharmacodynamic properties of statins: An update. Fundam. Clin. Pharmacol., 19(1): 117 - 125.

Toreson, H. and Eriksson, B.M. 1996. Determination of fluvastatin enantiomers and the racemate in human blood plasma by liquid chromatography and fluorometric detection. J. Chromatogr. A,729(1 - 2): 13 - 18.

Toreson, H. and Eriksson, B.M. 1997. Liquid chromatographic determination of fluvastatin and its enantiomers in blood plasma by automated solid-phase extraction. Chromatographia, 45:29 - 34. 
Um, S.Y., Jung, S.H., Jung, S.J., Kim, J.I., Chung, S.Y., Lee, H.J., Han, S.B., and Choi, S.O. 2006. Column-switching high-performance liquid chromatographic analysis of fluvastatin in rat plasma by direct injection. J. Pharm. Biomed.Anal., 41(4): 1458 - 1462. 